\title{
In-situ Polymerization of Styrene to Produce Polystyrene / Montmorillonite Nanocomposites
}

\author{
Lahouari Mrah* Rachid Megabar, Mohammed Belbachir \\ ${ }^{1}$ Laboratory of Polymer Chemical, Department of Chemistry, Faculty of Exact and Applied \\ Sciences, Oran University, Ahmed Benbella 1, 31100 Oran, Algeria
}

Received: 29th June 2015; Revised: 9 ${ }^{\text {th }}$ August 2015; Accepted: $15^{\text {th }}$ August 2015

\section{Abstract}

A reactive cationic surfactant cetyltrimethylammonium bromide (CTAB) was synthesized for intercalation of montmorillonite Mmt, a Maghnite type of clay. The pristine montmorillonite (Mmt) was obtained from Algerian plant with a cation exchange, Organophilic Mmt, was prepared by ion exchange between $\mathrm{Na}^{+}$ions in the clay. CTAB-intercalated Mmt particles were easily dispersed and swollen in styrene monomer, PS/Mmt-CTAB nanocomposites were synthesized via in-situ polymerization, in-situ polymerization, this method is based on the swelling of the silicate layers with the liquid polymer. The polymer composites were characterized using different techniques such as X-ray diffraction (XRD), The results were showed that, the basal space of the silicate layer increased, as determined by XRD, from 12.79 to $32.603 \AA$. Transmission electron microscopy (TEM) indicated that exfoliation of Mmt was achieved. In this current research, thermal gravimetric analysis (TGA) and force atomic microscopy (AFM) were also studied. (C) 2015 BCREC UNDIP. All rights reserved

Keywords: nanocomposites; Montmorillonite; in situ polymerization; force atomic microscopy (AFM); Xray diffraction

How to Cite: Mrah, L., Megabar, R., Belbachir, M. (2015). In-situ Polymerization of Styrene to Produce Polystyrene / Montmorillonite Nanocomposites. Bulletin of Chemical Reaction Engineering \& Catalysis, 10 (3): 249-255. (doi:10.9767/bcrec.10.3.8708.249-255)

Permalink/DOI: http://dx.doi.org/10.9767/bcrec.10.3.8708.249-255

\section{Introduction}

Since the inception 20 years ago by the Toyota group [1,2]. The synthesis of polymer/clay nanocomposites has been of considerable interest to the packaging and automotive industries. Polymer/clay nanocomposites are attractive and offer potential growth because of their wide range applications over traditional polymers [35]. These nanocomposites have proven to increase the mechanical [6], chemical and thermal properties [7]. Therefore these nanocompo-

\footnotetext{
* Corresponding author.
}

E mail: lmrah@yahoo.fr (L. Mrah)

Tel: +21341510057, Fax: +21341510057 sites generate a higher resistance to gas permeability [8-11], flammability [12,13]. The polymer/clay interactions within the matrix ultimately decide the morphology of the nancomposite. The morphologies of the clays in the nanocomposites have been cited as phase separated, intercalated or exfoliated [4,14,15]. Different synthesis procedures, in situ polymerization, solution blending and melt polymerization [16$20]$, have been used in order to obtain the different morphologies. The exfoliated or delaminated morphology is the preferred in order to maximize the effect of the clay in the matrix. The morphology of the nanocomposites has a direct effect on the mechanical, thermal and barrier properties [21,22]. Recently, another method used to obtain polymer/clay nanocom- 
posites has been emulsion polymerization [2326]. Emulsion polymerization offers a cleaner and "greener" alternative to the solvents used in in-situ polymerization and furthermore limits to the thermal degradation of the clay platelets that occurs in melt processing. The use of colloidal dispersions offers another broad range of applications for nanocomposite technology within the realm of coatings and paints [27]. Different groups [28-34], have used solid particles or in this case clay nanoparticles as stabilizers in emulsions in order to substitute surfactants. This process is commonly known as "Pickering emulsions [35]." Nanoclay platelets act as surfactants and are located at the liquidliquid interface [36], which causes a decrease in the interfacial energy.

The objective of this work is to develop polymer/clay nanocomposites as barrier membranes using dispersed and/or exfoliated phyllosilicates in a polymer matrix. Another property that may improve the dispersion of the layered materials is the interactions of the clays and the surfactants. The chemical modification of the clay surface may render improved polymer/clay or polymer/surfactant interactions yielding a different morphology in the matrix. In the present work, Algerian montmorillonite was organophilized and tested to prepare nanocomposite by melt blending. Montmorillonites, a class of inexpensive and noncorrosive. Our interest here in the influence of the formulation of the systems PS / clay on their structure as well as on their mechanical properties. The objective of this research was to synthesize high performance Polystyrene organoclay nanocomposites and to study the correlation between morphology and mechanical properties of the nanocomposites.

\section{Materials and Methods}

\subsection{Materials}

Polystyrene (PS) (grade 99\%) was used as purchased from Aldrich chemical. The cetyltrimethylammonium bromide were used as purchased from Aldrich chemical. RawMaghnite: Algerian montmorillonite clay which has been used as catalyst is supplied by a local company (ENOF Maghnia (Western of Algeria)).

\subsection{Preparation of Maghnite-Na ${ }^{+}$}

The bentonite was finely ground and sieved $(\leq 0.08 \mathrm{~mm})$, and then put in contact with the salt solution of $\mathrm{NaCl}(1 \mathrm{M})$, to swap the ions present in the clay for the cations $\mathrm{Na}^{+}[37,38]$.
It was then left under agitation for $12 \mathrm{~min}$. This operation was repeated 3 times, and then the clay was washed several times with distilled water to remove excess salt (precipitation of $\mathrm{Cl}$ - ions by $\mathrm{AgNO}_{3}$ ). The montmorillonitic suspensions were put in sedimentation graduated cylinders (two-liter test tubes) and kept at room temperature for 48 hours. Next, the suspension was siphoned by inhalation, by means of a pipette to recover the montmorillonite fraction containing particles of size around $2 \mu \mathrm{m}$. The remaining fraction was shaken again after each taking. This operation was repeated as many times as possible. Then, the montmorillonite was recovered and dried at $100{ }^{\circ} \mathrm{C}$, and finally crushed using a mortar.

\subsection{Organic Modification of Clay}

The montmorillonite was prepared and modified according to the protocol of Loïc Lepluart [39]. An amount of $10 \mathrm{ml}$ of $1 \mathrm{~N}$ hydrochloric acid was introduced into a one-liter volumetric flask, and then the volume was completed up to the mark with distilled water. After that, the resulting solution was poured into a conical flask fitted with a magnetic stirrer. This acid solution is put under a temperature of $80{ }^{\circ} \mathrm{C}$ used for the processes optimized via a heating magnetic agitator on a heating magnetic agitator. When the temperature became stable, $10^{-2}$ moles of cetyltrimethylammonium bromide were introduced into the mixture. After three hours of agitation at $80^{\circ} \mathrm{C}$, the amine is dissolved and ionized. Then, $5 \mathrm{~g}$ of sodium montmorillonite were added. After three hours of cationic exchange, the organophilic montmorillonite was recovered and rinsed six successive times with distilled water at $80^{\circ} \mathrm{C}$ to eliminate the inorganic cations. The efficiency of rinsing was checked by the addition of some drops of silver nitrate to the residue. The physisorbed alkylammonium ions were removed by a mixture of water and ethanol (50/50), heated to $60{ }^{\circ} \mathrm{C}$ beforehand. The organophilic montmorillonite obtained was then dried at $100{ }^{\circ} \mathrm{C}$, and then crushed. This is referred to as Montmorillonite-CTAB, throughout this study.

\subsection{Composites Preparation}

Four types of composites of different compositions (1, 3, 5 and $10 \mathrm{wt} \%$ MMT-CTAB ) were prepared by melt compounding at room temperature, the polymerization of styrene was carried out in tubes. Each tube contained a mixture of $10.0 \mathrm{~g}$ of styrene and an amount of montmorillonite-CTAB. The desired amount of 
cation exchanged MMT was dispersed in styrene monomer. The mixtures were held at under ambient temperature and stirred with a magnetic stirrer, the reaction was terminated by precipitating the PS/montmorillonite-CTAB. The nanocomposites was then denoted as PSNC1, PSNC3, PSNC5 and PSNC10 according to their containing clay [40].

\subsection{Characterization}

X-ray diffraction (XRD) analysis of Maghnite-CTAB was carried out using a Philips PW 1880 powder diffractometer $(\mathrm{Cu}-\mathrm{Ka}$ radiation, $\lambda$ $=1.541 \AA$ ). The topographic study of surfaces generally allows extracting information on the growth of thin films and especially of oxide layers. The near-field microscope "JSPM-4200" is calibrated in contact or tapping mode, according to the standard called the reference standard or metal grid, and using the magnetooptical Kerr effect. Remember that there is often a problem of resolution in atomic force microscopy (AFM), because of the size of the probe. Thermal gravimetric analysis was conducted on a Shimadzu TGA-51H analyser with $\mathrm{N}_{2}$. The phase morphologies of the nanocomposites were observed by transmission electron microscope (TEM), Philips CM 120, operating at an acceleration voltage of $120 \mathrm{KV}$.

\section{Results and Discussion}

Figure 1 (a) presents the diffractograms of both sodium montmorillonite MMT-Na and modified CTAB-montomorillonite MMT. MMT$\mathrm{Na}$ shows a peak at $2 \theta=6.95^{\circ}$, which corresponds to an interlayer distance $\mathrm{d}_{001}=12.97 \AA$. The addition of alkylammonium ions causes a displacement of the characteristic peak towards smaller angles, $2 \theta=2.46^{\circ}$, and therefore an in- crease in the interlayer distance, i.e. do01 $=$ $32.603 \AA$ for MMT-CTAB. This increase indicates the intercalation of alkyl ammonium ions in the interlayer galleries of MMT-Na, through a simple cation exchange. Note the appearance of a broad peak at $2 \theta \sim 5^{\circ}$, which may be due to a large scale organization of silicates.

Figure 1 (b) illustrates the diffraction patterns of X-ray PS / MMT with different concentrations of the clay content. As shown in Figure 1 (b), the peak is related to the clay has disappeared, suggesting that the silicate sheets are exfoliated. The weak signals for all the nanocomposites may suggest a partial delamination / exfoliation of the clay platelets in the matrix. Based on the disappearance or the decrease of intensity of XRD peaks, authors conclude the silicate is partially or completely exfoliated [41].

First, consider the situation where the flow of iron atoms is perpendicular to the surface. This surface is mainly composed of nanoscale iron islands, whose average diameter continuously increases. These are tetrahedral in shape, as evidenced by the discovery of the three facets. Using the auto-correlation function is the perfect way to analyze the average form and the spatial distribution of these islands. Whatever the thickness of the iron deposition is, the image obtained by autocorrelation presents the same profile; i.e. a circular central spot, characteristic of isotropic lateral distribution of islets. In Figure 2 (b), the images are compared. It is therefore clear that the grain size increases in the $\mathrm{Z}$ direction. This size along the $\mathrm{X}$ and $\mathrm{Y}$ axes is substantially equal to the training period, which indicates that a grain has a nonspherical shape.

This is probably due to the growth mechanism. This finding confirms the inhomogeneous

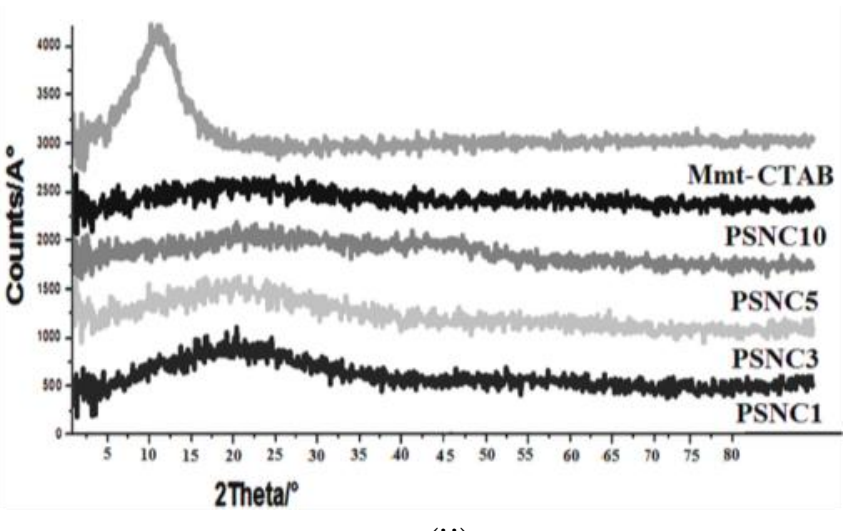

(ii)

Figure 1(b). XRD diffraction patterns: (i) MMT-Na (a) and MMT-CTAB (b); (ii) modified Mmt-CTAB and PSNC /clay series 


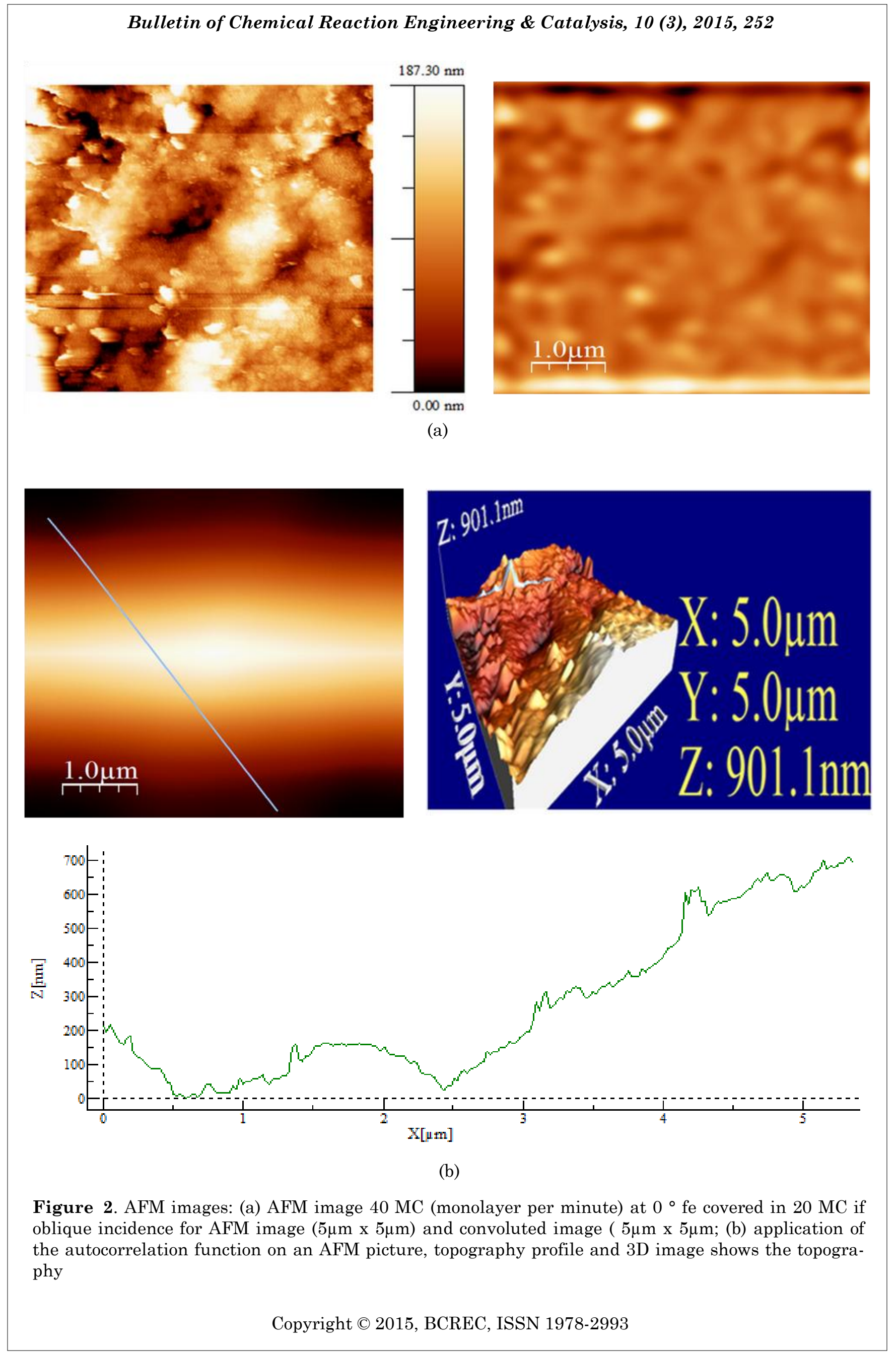


nature of PS / organophilic montmorillonite clay (CTAB) nanocomposites. The autocorrelation study allows determining the grain size.

The Mmt layers are randomly dispersed in polystyrene matrix. The more the mass percentage of montmorillonite $\mathrm{CTAB}$ is brought up, the more the capacity of the PS to exfoliate the clay. The morphology of the exfoliated Mmt also reflects the initial stacking arrangement of the layers of pristine clay particles. The exfoliation of Mmt in PS matrix may be attributed to the strong interaction between styrene and Mmt-CTAB.

The thermal decomposition behavior of PS and PS/ montmorillonite-CTAB nanocomposite is shown in Figure 4. Dietsche and Mulhaupt [42] also observed an improved thermal stability of acrylic composites using TGA technique. The strong fixation between inorganic surface and polymer is also considered to be due to the cooperative formation of ion induced dipole forces. This result is in agreement with those

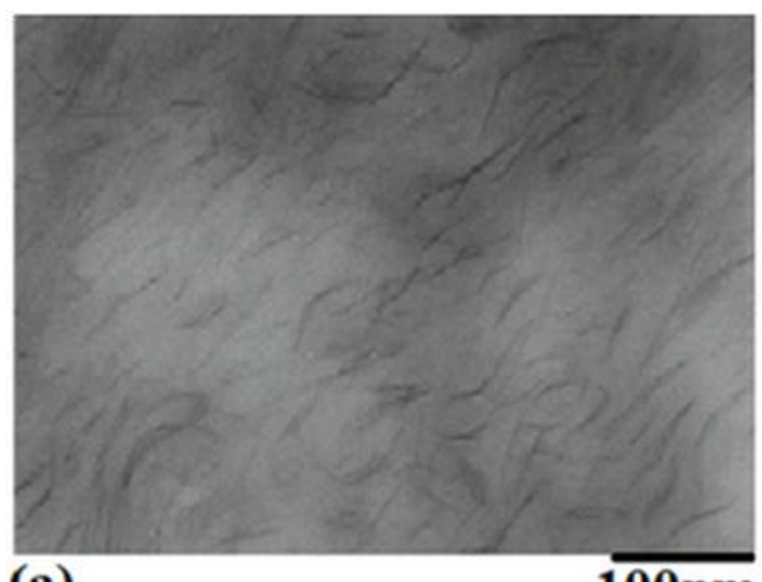

\section{(a)}

$\overline{100 n m}$

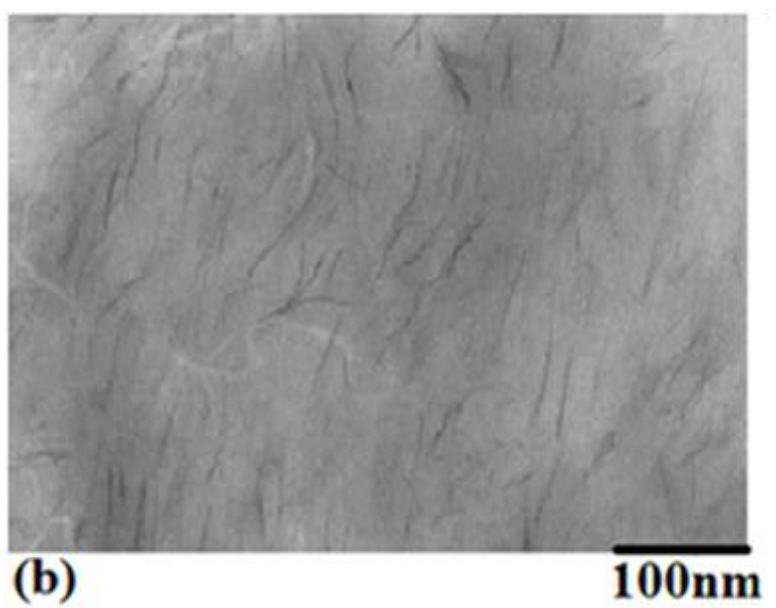

Figure 3. TEM micrograph of a nanocomposite PS / Mmt-CTAB containing 3 wt. $\%$ and 5 wt. $\%$ Mmt-CTAB evoked in similar research works or the others observe generally an increase of the degradation of nanocomposites because of good interaction polymer/clay or the plaques of clay shows above the curve (PS) of the products of degradation [43].

\section{Conclusions}

This study actually shows and confirms the inability of the PS matrix exfoliate the clay despite the organic treatment of montmorillonite. The PS / MMT-CTAB nanocomposite was synthesize by Polymerization in situ by using a surfactant polymerizable. The surfactant synthesizes is effective in the exfoliation of maghnite in the matrix of PS. The results of X-ray diffraction and transmission electron microscopy suggested that the nanocomposites provided exfoliated structures. The characterization by electronic microscopy to sweeping (AFM) of one PS / Mmt-CTAB 3.5\%, we show a dense grain structure in which the grains are very small and nanoscale. The grains are randomly distributed on all the surface of samples. Surfaces are not rather rough.

\section{Abbrevations}

PS: Poly Styrene

PSNC: Poly Styrene / Montmorillonite Nanocomposites

CTAB: Cetyltrimethylammonium Bromide

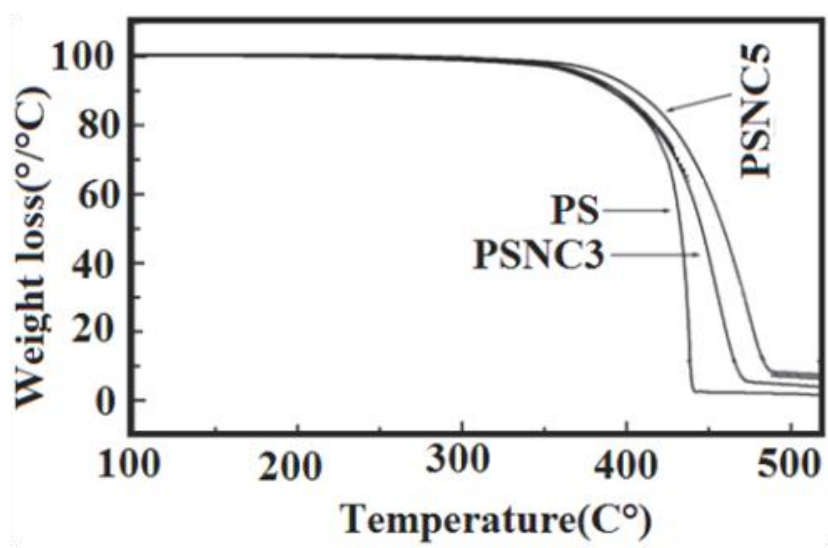

Figure 4. TGA thermograms of weight loss versus temperature: pure PS and Nanocomposite PS / montmorillonite-CTAB containing 3 wt.\% and 5 wt.\% Mmt-CTAB 


\section{References}

[1] Usuki, A., Kojima, Y., Kawasumi, M., Okada, A., Fukushima, Y., Kurauchi, T., Kamigaito, O. (1993). Synthesis of nylon 6-clay hybrid. Journal of Materials Research, 8(5): 11791184.

[2] Kojima, Y., Usuki, A., Kawasumi, M., Okada, A., Fukushima, Y., Kurauchi, T.,Kamigaito, O. (1993). Mechanical properties of nylon 6clay hybrid. Journal of Materials Research, 8(5): 1185-1189.

[3] Gao, F. (2004). Clay/polymer composites: the story. Materials Today, 7(11): 50-55.

[4] Tjong, S.C. (2006). Structural and mechanical properties of polymer nanocomposites. Materials Science and Engineering: R: Reports, 53(3-4): 73-198.

[5] Sinha Ray, S., and Okamoto, M. (2003). Polymer/layered silicate nanocomposites: a review from preparation to processing. Progress in Polymer Science, 28(11): 1539-1641.

[6] Wang, Z., and Pinnavaia, T.,J (1998). Nanolayer Reinforcement of Elastomeric Polyurethane. Chemistry of Materials, 10(12): 3769-3771.

[7] Yoon, P.J., Fornes, T.D., and Paul, D.R. (2002). Thermal expansion behavior of nylon 6 nanocomposites. Polymer, 43(25): $6727-$ 6741.

[8] Beyer, G. (2002). Nanocomposites: a new class of flame retardants for polymers. Plastics, Additives and Compounding, 4(10): 22-28

[9] Jeong, H.K., Krych, W., Ramanan, H., Nair, S., Marand, E., Tsapatsis M. (2004). Fabrication of Polymer/Selective-Flake Nanocomposite Membranes and Their Use in Gas Separation. Chemistry of Materials, 16(20): 38383845 .

[10] Ogasawara, T., Ishida, Y., Ishikawa, T., Aoki, T., Ogura, T. (2006). Helium gas permeability of montmorillonite/epoxy nanocomposites. Composites Part A: Applied Science and Manufacturing. 37(12): 2236-2240.

[11] Xu, B., Zheng, Q., Song, Y., Shangguan, Y. (2006) . Calculating barrier properties of polymer/clay nanocomposites: Effects of clay layers. Polymer. 47(8): 2904-2910.

[12] Cardenas, M.A., Garcia-Lopez, D., Gobernado- Mitre, I., Merino, J.C., Pastor, J.M., Martinez, J.De.D., Barbeta, J., Calveras, D.(2008). Mechanical and fire retardant proper-ties of EVA/clay/ATH nanocomposites - Effect of particle size and surface treatment of ATH filler. Polymer Degradation and Stability, 93(11): 2032-2037.

[13] Du, B., Guo, Z., Song, P., Liu, H., Fang, Z., $\mathrm{Wu}, \mathrm{Y}$. (2009). Flame retardant mechanism of organo-bentonite in polypropylene. Applied Clay Science, 45(3): 178-18.

[14] Alexandre, M., and Dubois, P. (2000). Polymer-layered silicate nanocomposites: preparation, properties and uses of a new class of materials. Material Science and Engineering $R$, 28(1-2): 1-63.

[15] Ray, S.S., Okamoto, K., and Okamoto, M. (2003). Structure-Property Relationship in Biodegradable Poly(butylene succinate)/Layered Silicate Nanocomposites. Macromolecules, 36(7): 2355-2367.

[16] Vaia, R.A., and Giannelis, E.P. (1997). Polymer Melt Intercalation in OrganicallyModified Layered Silicates: Model Predictions and Experiment. Macromolecules, 30: 80008009 .

[17] Nguyen, Q.T., and Baird, D.G. (2007). An improved technique for exfoliating and dispersing nanoclay particles into polymer matrices using supercritical carbon dioxide. Polymer, 48(23): 6923-6933.

[18] Zhang, Z., ZHAO, N., WEI, W., WU, D., SUN, Y. (2006). Synthesis and characterization of poly(butyl acrylate-co-methyl methacrylate) clay nanocomposites via emulsion polymerization. International Journal of Nanoscience, 5(2-3): 291-297.

[19] Herrera-Alonso, J.M., Marand, E., Little, J.C., Cox, S.S. (2009). Transport properties in polyurethane/clay nanocomposites as barrier materials: Effect of processing conditions. Membrane Science, 337(1-2): 208-214.

[20] Patel, S., Bandyopadhyay, A., Vijayabaskar, V., Bhowmick Anil.K. (2006). Effect of acrylic copolymer an terpolymer composition on the properties of in-situ polymer silica hybrid nanocomposites. Journal of Materials Science, 41(3): 927-936.

[21] Xu, Y., Brittain W.J., Xue, C., Eby, R.K. (2004). Effect of clay type on morphology and thermal stability of PMMA-clay nanocomposites prepared by thermal heterocoagulation method. Polymer, 45(11): 3735-3746.

[22] Yang, W.T., ko, T.H., Wang, S.C., Shih, P.I., Chang, M.J., Jiang, G.J. (2008). Preparation of polystyrene/clay nanocomposite by suspension and emulsion polymerization. Polymer Composites, 29(4): 409-414.

[23] Diaconu, G., Micusik, M., Bonnefond, A., Paulis, M., Leiza, J. R. (2009). Macroinitiator and macromonomer modified montmorillonite for the synthesis of acrylic/MMT nanocomposite latexes. Macromolecules, 42(9): 3316-3325.

[24] Donescu, D., Corobea, M.C., Uricanu, V., Radovici, C., Serban, S., Garea, S.A. (2007). Synthesis of polyvinylacetate-sodium montmorillonite hybrids by emulsion polymerization in 
the presence of anionic surfactants. Journal of Dispersion Science and Technology, 28(5): 671-679.

[25] Ruggerone, R., Christopher, J.G.P., Herrera, N.N., Bourgeat-Lami, Elodie, Månson, JanAnders E. (2009). Highly filled polystyrenelaponite nanocomposites prepared by emulsion polymerization. European Polymer Journal, 45(3): 621-629.

[26] Negrete-Herrera, N., Letoffe, J.M., Putaux, J.L., David, L., Bourgeat-Lami E. (2004). Aqueous dispersions of silane-functionalized laponite clay platelets. A first step toward the elaboration of water-based polymer/clay nanocomposites. Langmuir, 20(5): 1564-1571.

[27] Diaconu, G., Paulis, M., and Leiza, J.R. (2008.) Towards the synthesis of high solids Content waterborne poly(methyl methacrylate-co-butyl acrylate)/montmorillonite nanocomposites. Polymer, 48(10): 2444-2454.

[28] Zhang, J., Chen, K., and Zhao, H. (2008). PMMA colloid particles armored by clay Layers with PDMAEMA polymer brushes. Journal of Polymer Science A: Polymer Chemistry, 46(8): 2632-2639.'

[29] Bon, S.A.F., and Colver, P.J. (2007). Pickering miniemulsion polymerization using laponite clay as a stabilizer. Langmuir, 23(16): 83168322 .

[30] Guillot, S., Bergaya, F., de Azevedo, C., Warmont, F., Tranchant, Jean-François. (2009). Internally structred pickering emulsions stabilized by clay mineral particles. Journal of Colloid and Interface Science, 333(2): 563-569.

[31] Voorn, D.J., Ming, W., and van Herk, A.M. (2006). Polymer-clay nanocomposites latex particles by inverse pickering emulsion polymerization stabilized with hydrophobic montmorillonite platelets. Macromolecules, 39(6): 2137-2143.

[32] Wu, Y., Zhang, J., and Zhao, H. (2009). Functional colloidal particle stabilized by Layered silicate with hydrophilic face and hydrophobic polymer brushes. Journal of Polymer Science A: Polymer Chemistry, 47(6): 1535-1543.
[33] Ding, S., Liu, B., Zhang, C., Wu, Y., Xu, H. (2009). Amphiphilic mesoporous silica composite nanosheets. Journal of Materials Chemistry, 19: 3443-3448

[34] Cauvin, S., Colver, P.J., and Bon, S.A.F. (2005). Pickering stabilized miniemulsion polymerization: preparation of clay armored latexes. Macromolecules, 38(19): 7887-7889

[35] Pickering, S.U. (1907). Pickering: Emulsions. Journal of the Chemical Society Transactions, 91(23): 2001-2021.

[36] Binks, B.P. (2002). Particles as surfactants-similarities and differences. Current Opinion in Colloid \& Interface Science, 7(1-2): 21-41.

[37] Belbachir, M., Bensaoula, A. (2003). Composition and Method for Catalysis using Bentonites, US Patent 0069446 A1.

[38] Belbachir, M., Bensaoula, A. (2001). US Patent 6,274,527 B1.

[39] Le Pluart, Loïc. (2002). Nanocomposites, epoxyde, amine, montmorillonite : Role of Interactions on The formation, Morphology at Different Levels of Scale and Mechanical Properties of The Networks. PhD Thesis, National Institute of Applied Sciences, Lyon.

[40] Kherroub, D., Belbachir, M., Saad Lamouri. (2014). Nylon 6/clay nanocompositesvprepared with Algerian modified clay (12maghnite). Research on Chemical Intermediates, 41(8): 5217-5228.

[41] Liu, X., Wu, Q. (2001). PP/clay nanocomposites prepared by grafting melt Intercalation. Polymer, 42(25): 10013-10019.

[42] Dietsche, F., Mülhaupt, R. (1999). Thermal properties and flammability of acrylic nanocomposites based upon organophylic layered silicates, Polym. Bull. 43(4-5): 395-402.

[43] Abranyi, A., Szazdi, L., Pukanszky, B., Vancso, J., Pukanszky, B. (2006). Formation and Detection of Clay Network Structure in Poly(propylene)/Layered Silicate Nanocomposites, Macromol. Rapid Commun. 27(2): 132135. 